\title{
The Fokker-Planck equation, and stationary densities
}

\author{
Amir Aghamohammadi ${ }^{1}$ \& Mohammad Khorrami $^{2}$ \\ Department of Physics, Alzahra University, Tehran 1993891167, Iran.
}

\begin{abstract}
The most general local Markovian stochastic model is investigated, for which it is known that the evolution equation is the Fokker-Planck equation. Special cases are investigated where uncorrelated initial states remain uncorrelated. Finally, stochastic one-dimensional fields with local interactions are studied that have kink-solutions.
\end{abstract}

PACS numbers: 05.40.-a, 02.50.Ga

Keywords: Fokker-Planck, stationary solutions, kinks

\footnotetext{
${ }^{1}$ mohamadi@alzahra.ac.ir

${ }^{2}$ mamwad@mailaps.org
} 


\section{Introduction}

The Fokker-Planck equation was first used to describe the statistics of the Brownian motion of a particle in a fluid. This equation describes the time evolution of the probability density function of the position or the velocity of a Brownian particle. It is a differential equation, second order in the spatial variables and first order in time. There are also some studies on generalizations of the FokkerPlanck equation involving spatial derivatives of order higher than two [1-5]. Although these higher order equations are linear in the distribution function, these are sometimes referred to as nonlinear Fokker-Planck equations. There are also other generalizations of the Fokker-Planck equation [6,7]. In [8], it has been shown that any truncation of the generalized Fokker-Planck equation (the Kramers-Moyal expansion) is inconsistent, unless only terms of up to second order derivatives are kept.

Reaction-diffusion systems is a well-studied area. People have studied reactiondiffusion systems using analytical techniques, approximation methods, and simulations. A large fraction of exact results belong to low-dimensional (specially one-dimensional) systems, as solving low-dimensional systems should in principle be easier. Despite their simplicity, these systems exhibit a rich and rather nontrivial dynamical and stationary behavior. Studies on the models far from equilibrium have shown that there is a remarkably rich variety of critical phenomena [9]. There are some exact results involving kinks in one-dimensional reaction-diffusion models, as well as simulations and numeric results [10], and also mean field results [11]. Formation of localized shocks in one-dimensional driven diffusive systems with spatially homogeneous creation and annihilation of particles has been studied in [12]. Recently, in [13], the families of models with travelling wave solutions on a finite lattice have been presented. Shocks have been studied at both the macroscopic and the microscopic levels and there are efforts to understand how these macroscopic shocks originate from the microscopic dynamics [11]. Hydrodynamic limits have also been investigated. In a recent article both stationary and dynamical single-kinks on a one-dimensional lattice have been investigated [14]. It was done for both an infinite lattice and a finite lattice with boundaries. Static and dynamical phase transitions of these models have also been studied.

In such studies, one way is to investigate the system on a lattice and then take the limit of the results in the continuum. Another way is to write the equation in continuum from the beginning. In the latter case, one should write the master equation in continuum. This problem is addressed in this paper, and based on the results simple examples are studied.

The scheme of the paper is the following. In section 2, the Fokker-Planck equation and its generalizions are briefly reviewed, where it is known that if the evolution equation of the probability density is local in space, then the evolution equation involves no more than second spatial derivatives [8]. By locality, it is meant that the time derivative of probability density is expressible in terms of only a finite number of spatial derivatives of that density. This means that the evolution equation for the most general local stochastic Markovian models 
with is the Fokker-Planck equation. In section 3, this equation is generalized to the case of random fields. It is seen that there are special local interactions for which uncorrelated initial states remains uncorrelated. Finally, section 4 is devoted to some simple examples of stocahstic fields, admitting kink solutions.

\section{The Fokker-Planck equation}

To fix notation, let's briefly review the Fokker-Plank equation. Let $\mathbf{r}$ be a continuous ( $D$-dimensional) random variable, and $\psi(\mathbf{r}, t)$ be the corresponding probability density at the time $t$. One has

$$
\begin{aligned}
\psi(\mathbf{r}, t) & \geq 0 \\
\int \mathrm{d}^{D} r \psi(\mathbf{r}, t) & =1 .
\end{aligned}
$$

The evolution equation for this probability density is

$$
\psi(\mathbf{r}, t)=\int \mathrm{d}^{D} r^{\prime} U\left(\mathbf{r}, \mathbf{r}^{\prime} ; t, t^{\prime}\right) \psi\left(\mathbf{r}^{\prime}, t^{\prime}\right)
$$

where $U$ is the evolution operator with the following properties

$$
\begin{aligned}
U\left(\mathbf{r}, \mathbf{r}^{\prime} ; t, t^{\prime}\right) & \geq 0 \\
\int \mathrm{d}^{D} r U\left(\mathbf{r}, \mathbf{r}^{\prime} ; t, t^{\prime}\right) & =1, \\
U\left(\mathbf{r}, \mathbf{r}^{\prime} ; t, t\right) & =\delta\left(\mathbf{r}-\mathbf{r}^{\prime}\right) .
\end{aligned}
$$

Differentiating (2) with respect to $t$, one arrives at

$$
\frac{\partial \psi(\mathbf{r}, t)}{\partial t}=\int \mathrm{d}^{D} r^{\prime} \frac{\partial U\left(\mathbf{r}, \mathbf{r}^{\prime} ; t, t^{\prime}\right)}{\partial t} \psi\left(\mathbf{r}^{\prime}, t^{\prime}\right)
$$

or

$$
\frac{\partial \psi(\mathbf{r}, t)}{\partial t}=\int \mathrm{d}^{D} r^{\prime} H\left(\mathbf{r}, \mathbf{r}^{\prime} ; t\right) \psi\left(\mathbf{r}^{\prime}, t\right),
$$

where $H$ is defined through

$$
\frac{\partial U\left(\mathbf{r}, \mathbf{r}^{\prime} ; t, t^{\prime}\right)}{\partial t}=: \int \mathrm{d}^{D} r^{\prime \prime} H\left(\mathbf{r}, \mathbf{r}^{\prime \prime} ; t\right) U\left(\mathbf{r}^{\prime \prime}, \mathbf{r}^{\prime} ; t, t^{\prime}\right),
$$

or

$$
H\left(\mathbf{r}, \mathbf{r}^{\prime} ; t\right):=\left.\frac{\partial U\left(\mathbf{r}, \mathbf{r}^{\prime} ; t, t^{\prime}\right)}{\partial t}\right|_{t^{\prime}=t} .
$$

Using (3), one obtains

$$
\begin{aligned}
H\left(\mathbf{r}, \mathbf{r}^{\prime} ; t\right) & \geq 0, \quad \mathbf{r} \neq \mathbf{r}^{\prime} \\
\int \mathrm{d}^{D} r H\left(\mathbf{r}, \mathbf{r}^{\prime} ; t\right) & =0 .
\end{aligned}
$$


Introducing the the basis vectors $|\mathbf{r}\rangle$ and their dual basis covectors $\left\langle\mathbf{r}^{\prime}\right|$,

$$
\begin{aligned}
\left\langle\mathbf{r}^{\prime} \mid \mathbf{r}\right\rangle & =\delta\left(\mathbf{r}-\mathbf{r}^{\prime}\right), \\
\int \mathrm{d}^{D} r|\mathbf{r}\rangle\langle\mathbf{r}| & =1,
\end{aligned}
$$

one can write (1) to (8) in a more compact form. To do so, one defines the vector $|\psi\rangle$ and the operators $H$ and $U$ through

$$
\begin{aligned}
\psi(\mathbf{r}) & =\langle\mathbf{r} \mid \psi\rangle, \\
H\left(\mathbf{r}, \mathbf{r}^{\prime}\right) & =\left\langle\mathbf{r}|H| \mathbf{r}^{\prime}\right\rangle, \\
U\left(\mathbf{r}, \mathbf{r}^{\prime}\right) & =\left\langle\mathbf{r}|U| \mathbf{r}^{\prime}\right\rangle,
\end{aligned}
$$

and the covector $\langle\mathcal{S}|$ through

$$
\langle\mathcal{S}|:=\int \mathrm{d}^{D} r\langle\mathbf{r}| .
$$

One would have then

$$
\begin{aligned}
\langle\mathbf{r} \mid \psi\rangle & \geq 0, \\
\langle\mathcal{S} \mid \psi\rangle & =1, \\
|\psi(t)\rangle & =U\left(t, t^{\prime}\right)\left|\psi\left(t^{\prime}\right)\right\rangle, \\
\left\langle\mathbf{r}\left|U\left(t, t^{\prime}\right)\right| \mathbf{r}^{\prime}\right\rangle & \geq 0, \\
\langle\mathcal{S}| U\left(t, t^{\prime}\right) & =\langle\mathcal{S}|, \\
U(t, t) & =1, \\
\frac{\mathrm{d}}{\mathrm{d} t}|\psi(t)\rangle & =H(t)|\psi(t)\rangle, \\
\frac{\partial U\left(t, t^{\prime}\right)}{\partial t} & =H(t) U\left(t, t^{\prime}\right), \\
H(t) & :=\left.\frac{\partial U\left(t, t^{\prime}\right)}{\partial t}\right|_{t=t^{\prime}}, \\
\left\langle\mathbf{r}|H(t)| \mathbf{r}^{\prime}\right\rangle & \geq 0, \quad \mathbf{r} \neq \mathbf{r}^{\prime}, \\
\langle\mathcal{S}| H(t) & =0 .
\end{aligned}
$$

These are in fact continuum analogs of a system with discrete states, and can be readily obtained from that by suitably changing summations to integrations.

The evolution is said to be local (in space), if the time derivative of the density is expressible in terms of only a finite number of space derivatives of the density. We restrict ourselves to these cases. One then has

$$
\begin{aligned}
\frac{\partial \psi}{\partial t} & =\sum_{k=0}^{n}(-1)^{k} \partial_{i_{1}} \cdots \partial_{i_{k}}\left[f_{(k)}^{i_{1} \cdots i_{k}} \psi\right] \\
& =\sum_{k=0}^{n}(-1)^{k} g_{(k)}^{i_{1} \cdots i_{k}} \partial_{i_{1}} \cdots \partial_{i_{k}} \psi
\end{aligned}
$$


where $f^{i_{1} \cdots i_{k}}$ 's, and $g^{i_{1} \cdots i_{k}}$ 's are functions related to each other. This is equivalent to

$$
\begin{aligned}
H\left(\mathbf{r}, \mathbf{r}^{\prime} ; t\right) & =\sum_{k=0}^{n}(-1)^{k}\left[\partial_{i_{1}} \cdots \partial_{i_{k}} \delta\left(\mathbf{r}-\mathbf{r}^{\prime}\right)\right] f_{(k)}^{i_{1} \cdots i_{k}}\left(\mathbf{r}^{\prime}\right), \\
& =\sum_{k=0}^{n}(-1)^{k}\left[\partial_{i_{1}} \cdots \partial_{i_{k}} \delta\left(\mathbf{r}-\mathbf{r}^{\prime}\right)\right] g_{(k)}^{i_{1} \cdots i_{k}}(\mathbf{r}) .
\end{aligned}
$$

The fact that $\psi$ is a probability density imposes constraints on its evolution equation. If $\psi\left(\mathbf{r}_{0}, t_{0}\right)$ is zero, then $\psi$ attains a minimum at $\mathbf{r}_{0}$ and its first derivative vanishes at that point. As $\psi$ should remain nonnegative everywhere, one should have

$$
\frac{\partial \psi\left(\mathbf{r}_{0}, t_{0}\right)}{\partial t} \geq 0
$$

The initial condition $\psi\left(\mathbf{r}, t_{0}\right)$ is arbitrary, except that it should satisfy (1). So the derivatives of order more than two of $\psi$ with respect to $\mathbf{r}$ at $t=t_{0}$ and $\mathbf{r}=\mathbf{r}_{0}$ can attain arbitrary values. Suppose that of the derivatives (of order more than two) which appear in the right-hand side of (23), only one is nonvanishing and negative. If the absolute value of this derivative is large enough, then the righthand side of (23) becomes negative, which contradicts (25). So, one concludes that only derivatives of up to second order appear in the right-hand side of (23). This is discussed in [8]. Moreover, at a point where $\psi$ vanishes, the matrix of its second derivative should be positive semi-definite, as at that point $\psi$ attains its minimum. The matrix $g_{(2)}$ should be such that the time-derivative of $\psi$ be nonnegative at such a point. This means that $g_{(2)}$ should be positive semi-definite.

So, the eveolution equation for $\psi$ is

$$
\begin{aligned}
& \frac{\partial \psi}{\partial t}=f_{(0)} \psi-\partial_{i}\left[f_{(1)}^{i} \psi\right]+\partial_{i} \partial_{j}\left[f_{(2)}^{i j} \psi\right], \\
& \frac{\partial \psi}{\partial t}=g_{(0)} \psi-g_{(1)}^{i} \partial_{i} \psi+g_{(2)}^{i j} \partial_{i} \partial_{j} \psi .
\end{aligned}
$$

Integrating (26) on the whole space with $\psi(\mathbf{r}, t)=\delta\left(\mathbf{r}-\mathbf{r}_{0}\right)$, and using (1), one deduces that $f_{(0)}\left(\mathbf{r}_{0}\right)$ vanishes. As $\mathbf{r}_{0}$ is arbitrary, this means that

$$
f_{(0)}=0 \text {. }
$$

So (26) is simplified to

$$
\frac{\partial \psi}{\partial t}=-\partial_{i}\left[f_{(1)}^{i} \psi\right]+\partial_{i} \partial_{j}\left[f_{(2)}^{i j} \psi\right] .
$$

Comparing this with $(27)$, one obtains the relations between $f_{(k)}$ 's and $g_{(k)}$ 's:

$$
\begin{aligned}
0 & =g_{(0)}+\partial_{i} g_{(1)}^{i}+\partial_{i} \partial_{j} g_{(2)}^{i j}, \\
f_{(1)}^{i} & =g_{(1)}^{i}+2 \partial_{j} g_{(2)}^{i j}, \\
f_{(2)}^{i j} & =g_{(2)}^{i j} .
\end{aligned}
$$


Introducing the operators $\mathbf{R}$ and $\mathbf{P}$ through

$$
\begin{aligned}
R^{i}|\mathbf{r}\rangle & :=r^{i}|\mathbf{r}\rangle, \\
P_{i}|\mathbf{r}\rangle & :=\partial_{i}|\mathbf{r}\rangle,
\end{aligned}
$$

it is seen that

$$
\begin{aligned}
{\left[R^{i}, R^{j}\right] } & =0, \\
{\left[P_{i}, P_{j}\right] } & =0, \\
{\left[R^{i}, P_{j}\right] } & =\delta_{j}^{i},
\end{aligned}
$$

and

$$
\langle\mathcal{S}| P_{j}=0,
$$

and the Hamiltonian for the system can be written as

$$
\begin{aligned}
H & =P_{i} f_{(1)}^{i}(\mathbf{R})+P_{i} P_{j} f_{(2)}^{i j}(\mathbf{R}), \\
& =-\left[\partial_{i} g_{(1)}^{i}+\partial_{i} \partial_{j} g_{(2)}^{i j}\right](\mathbf{R})+g_{(1)}^{i}(\mathbf{R}) P_{i}+g_{(2)}^{i j}(\mathbf{R}) P_{i} P_{j},
\end{aligned}
$$

\section{Random fields}

Generalization of the arguments of the previous section to the case of fields

is quite straight forward. A random field is a collection of random variables defined at each point of the space. One only needs to consider the following correspondences.

$$
\begin{aligned}
|\mathbf{r}\rangle & \rightarrow|\varrho\rangle, \\
R^{i} & \rightarrow \rho(\mathbf{x}), \\
P_{i} & \rightarrow \Pi(\mathbf{x}), \\
\sum_{i} & \rightarrow \int \mathrm{d}^{n} x, \\
\partial_{i} & \rightarrow \frac{\delta}{\delta \varrho(\mathbf{x})}, \\
\int \mathrm{d}^{D} r & \rightarrow \int D \varrho,
\end{aligned}
$$


where $\rho$ is the random field operator, and one has

$$
\begin{aligned}
\rho(\mathbf{x})|\varrho\rangle & =\varrho(\mathbf{x})|\varrho\rangle, \\
\Pi(\mathbf{x})|\varrho\rangle & =\frac{\delta}{\delta \varrho(\mathbf{x})}|\varrho\rangle, \\
{[\rho(\mathbf{x}), \rho(\mathbf{y})] } & =0, \\
{[\Pi(\mathbf{x}), \Pi(\mathbf{y})] } & =0, \\
{[\rho(\mathbf{x}), \Pi(\mathbf{y})] } & =\delta(\mathbf{x}-\mathbf{y}), \\
\left\langle\varrho \mid \varrho^{\prime}\right\rangle & =\delta\left(\varrho-\varrho^{\prime}\right), \\
\langle\mathcal{S}| & :=\int D \varrho\langle\varrho|, \\
\langle\mathcal{S}| \Pi(\mathbf{x}) & =0 .
\end{aligned}
$$

It then follows that (34) is changed to

$$
H=\int \mathrm{d}^{n} x \Pi(\mathbf{x}) f_{(1)}[\mathbf{x} ; \rho]+\int \mathrm{d}^{n} x \mathrm{~d}^{n} y \Pi(\mathbf{x}) \Pi(\mathbf{y}) f_{(2)}[\mathbf{x}, \mathbf{y} ; \rho],
$$

and $f_{(2)}$ is positive semi-definite, that is, one has for arbitrary $h$ and $\varrho$,

$$
\int \mathrm{d}^{n} x \mathrm{~d}^{n} y h(\mathbf{x}) h(\mathbf{y}) f_{(2)}[\mathbf{x}, \mathbf{y} ; \varrho] \geq 0 .
$$

The evolution equation for $\psi$ (the probability distribution functional for $\rho$ ) is given analogues to (26):

$$
\begin{aligned}
\frac{\partial \psi}{\partial t}= & -\int \mathrm{d}^{n} x \frac{\delta}{\delta \varrho(\mathbf{x})}\left(f_{(1)}[\mathbf{x} ; \varrho] \psi[\varrho]\right) \\
& +\int \mathrm{d}^{n} x \mathrm{~d}^{n} y \frac{\delta^{2}}{\delta \varrho(\mathbf{x}) \delta \varrho(\mathbf{y})}\left(f_{(2)}[\mathbf{x}, \mathbf{y} ; \varrho] \psi[\varrho]\right) .
\end{aligned}
$$

One may also obtain the evolution equation for the expectation value of the field $(\langle\rho(\mathbf{z})\rangle=\langle\mathcal{S}| \rho(\mathbf{x}|\psi\rangle)$. One has

$$
\begin{aligned}
\frac{\partial \rho(\mathbf{z})}{\partial t} & =[\rho(\mathbf{z}), H], \\
& =f_{(1)}[\mathbf{z} ; \rho]+2 \int \mathrm{d}^{n} x \Pi(\mathbf{x}) f_{(2)}[\mathbf{x}, \mathbf{z} ; \rho],
\end{aligned}
$$

from which

$$
\frac{\partial}{\partial t}\langle\rho(\mathbf{z})\rangle=\left\langle f_{(1)}[\mathbf{z} ; \rho]\right\rangle
$$

It is seen that $f_{2}$ has no explicit contribution in the expectation value. It does, however, have implicit contribution through $\rho$. A special case is $f_{2}=0$. It can be shown then that if $f_{(1)}$ is a local functional of $\rho$, that is if $f_{(1)}[\mathbf{z} ; \rho]$ depends on 
only $\rho$ and a finite number of its derivatives in $\mathbf{z}$, then if the system is initially uncorrelated in space,

$$
\langle\rho(\mathbf{x}) \rho(\mathbf{y})\rangle=\langle\rho(\mathbf{x})\rangle\langle\rho(\mathbf{y})\rangle, \quad \mathbf{x} \neq \mathbf{y}
$$

then it remains uncorrelated in space. For a system initially uncorrelated, and for $\mathbf{x} \neq \mathbf{y}$, one has initially

$$
\begin{aligned}
\frac{\partial}{\partial t}\langle\rho(\mathbf{x}) \rho(\mathbf{y})\rangle & =\left\langle f_{(1)}[\mathbf{x} ; \rho] \rho(\mathbf{y})\right\rangle+\left\langle\rho(\mathbf{x}) f_{(1)}[\mathbf{y} ; \rho]\right\rangle, \\
& =\left\langle f_{(1)}[\mathbf{x} ; \rho]\right\rangle\langle\rho(\mathbf{y})\rangle+\langle\rho(\mathbf{x})\rangle\left\langle f_{(1)}[\mathbf{y} ; \rho]\right\rangle, \\
& =\left[\frac{\partial}{\partial t}\langle\rho(\mathbf{x})\rangle\right]\langle\rho(\mathbf{y})\rangle+\langle\rho(\mathbf{x})\rangle\left[\frac{\partial}{\partial t}\langle\rho(\mathbf{x})\rangle\right],
\end{aligned}
$$

which shows that initially

$$
\frac{\partial}{\partial t}[\langle\rho(\mathbf{x}) \rho(\mathbf{y})\rangle-\langle\rho(\mathbf{x})\rangle\langle\rho(\mathbf{y})\rangle]=0 .
$$

So the system remains uncorrelated.

Applying the approximation

$$
\left\langle f_{(1)}[\mathbf{x}, \rho]\right\rangle \approx f_{(1)}[\mathbf{x},\langle\rho\rangle],
$$

the evolution equation for the expectation value of $\rho$ becomes

$$
\frac{\partial}{\partial t}\langle\rho(\mathbf{x})\rangle=f_{(1)}[\mathbf{x} ;\langle\rho\rangle] .
$$

If $f_{(1)}$ is local, then the right-hand side of the above equation is the action of a differential operator on $\langle\rho(\mathbf{x})\rangle$.

\section{Simple examples}

Consider a one-dimensional system for which $f_{(1)}$ is local, apply the approximation (45), and assume that

$$
f_{(1)}[x, \rho]=\frac{\partial^{2} \rho}{\partial x^{2}}+F[\rho(x)] .
$$

Then the evolution equation for the expectation of $\rho(x)$ is

$$
\frac{\partial\langle\rho(x, t)\rangle}{\partial t}=\frac{\partial^{2}\langle\rho(x, t)\rangle}{\partial x^{2}}+F[\langle\rho(x, t)\rangle] .
$$

Of special interest are the time-dependent solutions to this equation:

$$
\frac{\mathrm{d}^{2} u(x)}{\mathrm{d} x^{2}}+F[u(x)]=0,
$$


where

$$
u(x):=\langle\rho(x)\rangle .
$$

Introducing the potential function $V$ through

$$
\frac{\mathrm{d} V}{\mathrm{~d} u}:=F(u),
$$

one can once integrate (49) to obtain

$$
\frac{1}{2}\left(\frac{\mathrm{d} u}{\mathrm{~d} x}\right)^{2}+V(u)=\text { const. }
$$

An obvious solution to this, or (49), is

$$
u(x)=u_{0},
$$

where $u_{0}$ is z zero of $F$. A kink solution is a solution which tends to constant values at infinity, but the value at $+\infty$ is different from the value at $-\infty$ :

$$
\lim _{x \rightarrow \pm \infty} u(x)=u_{ \pm}, \quad u_{+} \neq u_{-} .
$$

For such a solution to exist, one should have

$$
\begin{aligned}
& V\left(u_{-}\right)=V\left(u_{+}\right), \\
& \left.\frac{\mathrm{d} V}{\mathrm{~d} u}\right|_{u_{ \pm}}=0,
\end{aligned}
$$

$V$ should be less than $V\left(u_{-}\right)=V\left(u_{+}\right)$if $u$ is between $u_{-}$and $u_{+}$. A simple example is

$$
V(u)=-\alpha\left(u-u_{-}\right)^{2}\left(u-u_{+}\right)^{2},
$$

where $u_{ \pm}$and $\alpha$ are constants and $\alpha$ is positive.

A multi-kink solution is a solution that tends to constant (different) values at plus and minus infinity, and is constant (or approximately constant) in some other intervals as well. Such solutions exist if there are some points $u_{i}$ between $u_{-}$and $u_{+}$, where the potential $V$ becomes nearly equal to $V\left(u_{-}\right)=V\left(u_{+}\right)$. One then has a solution which tends to constants at infinity, and is nearly equal to $u_{i}$ and nearly constant for a large region. An example is

$$
V(u)=-\left[\alpha+\beta\left(u-u_{0}\right)^{2}\right]\left(u-u_{-}\right)^{2}\left(u-u_{+}\right)^{2},
$$

where $u_{ \pm}, \alpha$, and $\beta$ are constants, $\alpha$ and $\beta$ are positive, and $u_{0}$ is between $u_{-}$ and $u_{+}$. If $\alpha$ is small, then there is an approximately double-kink solution.

A special case is when $V\left(u_{0}\right)$ is exactly equal to $V\left(u_{ \pm}\right)$. In this case, if $V$ has second derivative in $u_{0}$, then the double-kink solution disappears and two distinct single-kink solutions emerge: one with between $u_{-}$and $u_{0}$, the other between $u_{0}$ and $u_{+}$. However, if the first derivative of $V$ near $u_{0}$ tends to zero 
like $\left|u-u_{0}\right|^{\gamma}$ with $\gamma<1$, then there is a solution with a flat part at $u_{0}$ of a finite width. An example is

$$
V(u)=-\beta\left|u-u_{0}\right|^{1+\gamma}\left(u-u_{-}\right)^{2}\left(u-u_{+}\right)^{2},
$$

where $0<\gamma<1$.

These are stationary solutions. It is easy to see that a stationary solution is readily converted to a travelling solution using a Galilean transformation. This transformation only changes the time-derivative $\frac{\partial}{\partial t}$ to $\frac{\partial}{\partial t}+\mathbf{v} \cdot \nabla$, where $\mathbf{v}$ is the corresponding velocity parameter, and is in fact the velocity of the travelling solution. 


\section{References}

[1] N. G. van Kampen; Can. J. Phys. 39 (1961) 551.

[2] J. L. Lebowitz \& E. Rubin; Phys. Rev. (1964) 2381.

[3] P. Resibois \& H. T. Davis; Physica 30 (1964) 1077

[4] H. Mori, H. Fujisaka, \& H. Shigematsu; Prog. Theor. Phys. 51 (1974) 109.

[5] A. V. Plyukhin; cond-math/0501676.

[6] V. E. Tarasov; Chaos 2 (2005) 023102.

[7] P. H. Chavanis; cond-math/0504716.

[8] R. F. Pawula; Phys. Rev. 162 (1967) 186.

[9] G. M. Schütz; "Exactly solvable models for many-body systems far from equilibrium" in "Phase transitions and critical phenomena, vol. 19", C. Domb \& J. Lebowitz (eds.) (Academic Press, London, 2000).

[10] F.H. Jafarpour; cond-mat/0403099.

[11] M. Paessens \& G. M. Schütz; cond-mat/0406591.

[12] V. Popkov, A. Rakos, R.D. Willmann, A.B. Kolomeisky, \& G.M. Schütz; Phys. Rev. E67 (2003) 066117.

[13] K. Krebs, F.H. Jafarpour, \& G.M. Schütz; New J. Phys. 5 (2003) 145.1.

[14] M. Arabsalmani \& A. Aghamohammadi; Phys. Rev. E74 (2006) 011107. 